\title{
APUNTES PARA LA EVALUACIÓN DE LA ACTIVIDAD DE LAS SOCIEDADES MUSICALES EN ESPAÑA
} $(1921-25)$

\author{
Gemma PÉREZ ZALDUONDO
}

Adolfo Salazar, en 1930, definió las sociedades filarmónicas como "los principales órganos de difusión de la música en España", indicando así la innegable importancia de las mismas para el conocimiento de la evolución musical de comienzos del Siglo XX en nuestro país. A pesar de ello, existen escasos aportes documentales sobre el tema².

En el presente artículo pretendemos ofrecer y analizar datos parciales sobre la actividad de la Sociedad Filarmónica de Madrid y la Sociedad Sevillana de Conciertos, junto a otros pertenecientes a asociaciones musicales de diversa índole ${ }^{3}$. Todos los datos corresponden a las temporadas concertísticas de los años 1921 a $1925^{4}$, período en el que se produjeron acontecimientos tan significativos como el estreno de El Retablo de Maese Pedro en ambas Sociedades y las visitas de Wanda Landowska y Arthur Rubinstein a Sevilla.

Hemos centrado nuestro análisis en: la programación de las temporadas de conciertos; los intérpretes contratados; la clasificación estilística del repertorio; la presencia de la música española y la novedad de las obras ejecutadas.

1. Salazar, Adolfo, La música Contemporánea en España, Madrid, Ediciones La Nave, 1930. Ed. facsímil: Oviedo, Ethos Música, 1982, p. 304.

2. Ver CaSARes, Emilio, "La música española hasta 1939, o la restauración musical", en Actas del Congreso Internacional «España en la Música de Occidente». Madrid, Ministerio de Cultura, 1987, vol II, pp. 261-322. Torres, Jacinto, "Orquestas y sociedades (1900-1939)", op. cit., pp. 351-368. Siemens Hernández, Lothar, Historia de la Sociedad Filarmónica de Las Palmas y de su Orquesta y sus Maestros, Las Palmas de Gran Canaria, Sociedad Filarmónica, 1995.

3. Sociedades Filarmónicas de Valencia y Vigo, Asociación de Cultura Musical de Granada, Associació de Música "Da Camera" de Barcelona, Asociación de Conciertos de Reus.

4. Las fuentes consultadas proceden de los fonćos del Archivo Manuel de Falla de Granada y programas de conciertos de nuestro archivo particular. 


\section{Programación de las temporadas}

El factor determinante en la programación de las temporadas de conciertos era el potencial económico de las sociedades, dependiente, a su vez, de la cuantía de las cuotas y el número de socios. Estas circunstancias determinaban no sólo la contratación de intérpretes, sino, lo que es más importante, la programación del repertorio. Es evidente que los conciertos orquestales requerían un gasto mayor, cuya medida es posible seguir en los presupuestos de cada uno de los meses de la Sociedad Sevillana de Conciertos durante la temporada 1922-23: el correspondiente al mes de abril, en que se ofrecieron dos audiciones de la Orquesta Rusa de Balalaikas, requirió un desembolso de 6.366,45 ptas, y el de mayo, con tres de la Orquesta Sinfónica, 10.223,65 ptas ${ }^{5}$. Ambas cantidades suman el equivalente al total del resto de la temporada (51.802,85 ptas), si excluimos los dos conciertos de óperas cómicas que inauguraron las sesiones y la interpretación de El Retablo en el mes de marzo, acontecimientos estelares del período que supusieron 7.315,80 ptas y $9.726,10$ ptas respectivamente.

A los gastos en concepto de honorarios ${ }^{6}$ es necesario sumar los correspondientes al alquiler del Teatro San Fernando, donde tenían lugar las sesiones y cuya capacidad determinaba el número de socios. Como consecuencia, existían problemas económicos que, en meses determinados, hicieron que la Junta Directiva de la Sociedad Sevillana tuviera que recurrir al "peculio particular" para afrontarlos. No es de extrañar, por lo tanto, que en la Junta General Ordinaria de 25 de junio de 1923, fecha en la que el número de los asociados era 818 con una contribución de cinco ptas al mes, se propusiera el aumento de esta última a siete ptas, aunque finalmente quedó acordado que los socios fundadores o numerarios aportarían para el sostenimiento de la Sociedad una cuota mensual de seis ptas ${ }^{7}$.

Otro factor importante, en este caso referente a la gestión de las juntas directivas, es la adecuación de la temporada real a las previsiones hechas a comienzos de la misma. En el período 1923-24 de la Sociedad Filarmónica de Madrid, de los dieciocho conciertos anunciados ${ }^{8}$, cuatro estaban sin determinar ${ }^{9}$. Posteriormente, se añadió idéntico número ${ }^{10}$, mientras que el programa restante transcurrió sin cambios, con excepción de dos fechas puntuales ${ }^{11}$.

5. Memoria presentada por la Junta Directiva a los Socios en la Junta General Ordinaria de 25 de junio de 1923. Se especifican los presupuestos mensuales de la Sociedad Sevillana de Conciertos, con relación de gastos e ingresos así como el cachet de los intérpretes.

6. Wanda Landowska, 2.000 ptas; Vera Janacopulos, 1.500 ptas; Orquesta Sinfónica de Madrid: 7.500 ptas. Artistas y orquesta de las óperas de inauguración, 5.300; Cuarteto Inglés, 1.800; Lola Schlepianoff, 500; Quinteto Wendling de Stuttgart, 2.300; Zadora, 800; Bildstine, 900; Paul Kochanski, 2.100; Tomás Teran, 1.200; Orquesta Rusa de Balalaikas, 5.000; Herminia Peñaranda, 500; Camino Bejar, 550 ptas. Alquiler del Teatro San Fernando, de octubre a mayo, 4.728,50 ptas.

7. En Memoria presentada..., ya citada.

8. Avance de programa (fondos del Archivo Manuel de Falla).

9. Los correspondientes al mes de noviembre, que finalmente corrieron a cargo de Jeanne Marie Darré (piano), Suzanne Cesbron-Viseur (soprano) y Daniel Montorio (piano de acompañamiento), y al mes de febrero, del Cuarteto Zimmer.

10. Tres de los cuales se celebraron en el mes de febrero, todos ellos a cargo del pianista Télémaque Lambrino. El cuarto consistió en un recital de canto y piano, en el mes de marzo, a cargo de Lilly Hafgren y Janos Baranyi.

11. Diciembre: dos del violinista Zoltan Székely y del pianista Fernando Ember, y dos del Cuarteto Vocal de Hamburgo. Enero: tres de José Iturbi y uno de la soprano Dagmara Renina y el pianista Daniel Montorio, si bien este último cambió de fecha. Marzo: Orquesta Filarmónica de Madrid, prevista para el día 15, se desplazó al 28. En el mes de abril tenían comprometidas sus interpretaciones la violonchelista Guillermina Suggia, así como Bronislaw Hubermann y Siegfried Schultz, estos últimos en los días 
Por lo que respecta al número de audiciones, los datos obtenidos ratifican la afirmación de Adolfo Salazar, que indica el gran número de ellas que se celebraban en el ámbito de las sociedades filarmónicas $^{12}$. En el caso de la madrileña, su programación contemplaba una temporada reducida en el tiempo, de noviembre a abril, calendario compartido con el resto de entidades musicales de la capital, ya que José Subirá, al resumir el año 1924, señala:

"hubo una hipertrofia de recitales y conciertos desde enero a mayo y en la segunda mitad del otoño, contrastando con ella la paralización sufrida en los restantes meses."

El número de sesiones organizadas por la Filarmónica de Madrid en la temporada 1923-1924 asciende a veintiuna, desde el número 370 de la Sociedad al 391, ambos inclusive, la cifra mayor en relación a períodos inmediatamente anteriores y posteriores, puesto que se eleva desde diecisiete a veinte en las comprendidas entre 1921-22 y 1922-23 respectivamente, para volver al punto medio de estos años, veinte, en la correspondiente a $1924-25^{14}$.

De forma similar, la Sociedad Sevillana de Conciertos organizó, desde octubre de 1922 a mayo de 1923, diecinueve sesiones musicales con alguna desproporción en su periodicidad, ya que en febrero se ofreció uno, mientras que en mayo fueron tres los interpretados. De cualquier modo, la cifra total sobrepasa, también en este caso, la previsión inicial, esto es, la programación de dos conciertos mensuales según indica la Memoria de junio 1923.

\section{Intérpretes}

La contratación de los intérpretes se realizaba para dos actuaciones sucesivas. Además, con frecuencia dichas contrataciones suponían estancias de los mismos artistas en diversas ciudades españolas, es decir, dentro de giras, como la de Vera Janacopulos y Lola Schlepianoff, a quienes encontramos en la Sociedad Sevillana y en la Filarmónica de Madrid en diciembre de 1922. Este mismo hecho se puede observar en los programas de mano editados por la Sociedad madrileña: En el correspondiente al pianista Janos Baranyi, se alude al éxito que "recientemente" ha alcanzado en Barcelona; Respecto al Cuarteto Zimmer, se explica el incremento de sus conciertos en España debido también a los triunfos recogidos en el curso anterior; De Emmy

21, 23 y, junto a la Orquesta Filarmónica, el 26. Hemos podido verificar la interpretación del correspondiente al día 21, no así los dos últimos ni el de Guillermina Suggia, a cuyos programas de mano no hemos tenido acceso. Sin embargo, dado que Bronislaw Hubermann y Siegried Schultz ofrecieron el primero de los programados, suponemos que los dos restantes se desarrollarían con normalidad.

12. Salazar, Adolfo, op. cit., p. 304.

13. Subira, José, "El Año Musical", Hispania, enero de 1925, p. 30.

14. Desconocemos la fecha de apertura de la temporada 1921-22, pero el último concierto se ofreció el 1 de mayo de 1922. Su Programa de mano, contrariamente a lo acostumbrado en los editados por esta Sociedad, no indica el programa del siguiente, sino que señala la fecha de la Junta Ordinaria. Además, el número de concierto de la Sociedad, 349, es inmediatamente anterior al primero de la temporada posterior. En 1922-23 los conciertos ofrecidos se iniciaron con el 350 de la Sociedad, el 27 de noviembre de 1922 , finalizando con el 370. La temporada 1923-24 recorre desde el número 370 de la Sociedad hasta el 391. En la temporada 1924-1925 los números de los conciertos comienzan en el 392 y finalizan con el 412. 
Krüger, que actuó en la Sociedad en enero de 1925, se da noticia de su reciente actuación en el Teatro Real de Madrid, etc.

La consecuencia lógica de este régimen de contratación es la presencia de los mismos intérpretes y repertorios por toda la geografía nacional, cuestión a la que se refiere Adolfo Salazar en 1924:

"Sin embargo, salvo Barcelona que tiene una vida musical propia, el resto de la nación apenas disfruta, salvo muy señaladas excepciones - casi siempre, artistas locales-, de manifestaciones de este arte que no hayan tenido lugar también en la capital. Dado el régimen cooperativo por el que desde hace años se rigen la Sociedades Filarmónicas - un verdadero «Sindicato de Filarmónicas», con todas sus ventajas e inconvenientes-, si hoy existiese todavía una Revista general de música, las crónicas de los corresponsales de provincias serían, por turno, un desfile de opiniones sobre el mismo cuarteto, o el mismo violinista, a través de Burgos, Palencia, Guadalajara, Gijón, Oviedo, Avilés, Cartagena y así sucesivamente."15

En cuanto a la procedencia de los artistas, destaca la exigua presencia de intérpretes nacionales que, no obstante, son objeto de gran atención por parte de la crítica ${ }^{16}$. En la temporada 1923-24 de la Filarmónica de Madrid, se limitó a la de Joaquín Turina, cuya presencia puntual fue considerada como un "honor" para la Sociedad; Daniel Montorio como pianista de acompañamiento, y José Iturbi, que ya había actuado en la Sociedad madrileña el año anterior ${ }^{17}$ y había rescindido su contrato con la Sevillana de Conciertos para dos audiciones en diciembre de 1922. En la temporada siguiente encontramos únicamente los nombres de José Cubiles acompañando la interpretación de Emmy Krüger; Antonio Fernández Bordás y el pianista Antonio Lucas Moreno $^{18}$, cuya excelente técnica había sido señalada con anterioridad por Rogelio Villar ${ }^{19}$.

La procedencia de los intérpretes de la Filarmónica de Madrid es, por lo tanto, marcadamente internacional. Destacan especialmente los franceses, no sólo por el número de ellos que trabajan para la Sociedad, sino por la frecuencia con la que lo hacen: En abril de 1922 Edouard Risler había actuado en 55 ocasiones a lo largo de trece temporadas anteriores; El mismo año Magda Tagliaferro se presenta por cuarta vez ${ }^{20}$. Sin embargo, una de las figuras

15. Salazar, Adolfo, "El año musical", El Sol, 1 de enero de 1924, p. 13.

16. Adolfo Salazar dedica críticas, entre otros, a: Pilar Bayona y el Trio Hispania, que actuaron en la Filarmónica de Madrid en enero de 1923 (Ver Salazar, Adolfo, "La Vida Musical", El Sol, 25 de enero de 1923); Andrés Segovia, en la Asociación de Cultura Musical de Madrid en diciembre de 1924 (op. cit, 20 de diciembre de 1924); José Cubiles en el Teatro de la Comedia en junio de 1926 (op. cit, 10 de junio de 1926).

17. 25 de noviembre de 1922 (Concierto I, 350 de la Sociedad). 27 de noviembre de 1922 (Concierto II, 351 de la Sociedad). 29 de noviembre de 1922 (junto a la Orquesta Filarmónica bajo la dirección del maestro Pérez Casas).

18. Antonio Fernández Bordás (1870-1950) fue profesor de violín y música de cámara del Conservatorio de Madrid, del que también era Director en estos años, y primer violín de la Real Capilla. José Cubiles ingresaría en el Conservatorio madrileño, como Profesor numerario, en 1926, y Antonio Lucas Moreno en 1930.

19. Villar, Rogelio, "Los Conciertos. Jean Aubry", Nuevo Mundo, 7 de mayo de 1920.

20. Edouard Risler (Baden-Baden 1873-París 1929) fue uno de los grandes pianistas franceses, abierto tanto a la música de su tiempo como a la herencia romántica alemana, y ejerció gran influencia sobre otros pianistas, como Alfred Cortot. A partir de 1923 fue profesor del Conservatorio de París. Chabrier y Granados le dedicaron obras y estrenó partituras de Dukas, Fauré, Chausson, Reynaldo Hahn y Samazeuilh. En la temporada 1921-22 de la Sociedad Filarmónica de Madrid, Risler dirigió la Orquesta Filarmónica en su segunda actuación. Magda Tagliaferro, pianista francesa de origen brasileño (Petrópolis, Brasil, 1894-Río de Janeiro 1986), fue alumna de Cortot, Thibaud y Casals. Realizó una gira con Gabriel Fauré y con el Cuarteto Capet. Le dedicaron obras Reynaldo Hahn, Villa-Lobos y Migot (ver PÂRIS, Alain, Diccionario de intérpretes y de la interpretación musical en el siglo XX, Madrid, Turner, 1989) 
estelares de la temporada 1921-22 fue sin duda Vera Janacopulos, a la que Adolfo Salazar se refiere como:

"intérprete eminentísima, consagrada en el mundo musical extranjero como una de las primeras intérpretes de la música moderna",21.

En el período 1923-24 la presencia de los intérpretes franceses disminuye a la visita de Jeanne Marie Darré y Suzanne Cesbron-Viseur, la primera de las cuales había debutado en París en 1920 y en esta época veía materializarse su carrera. En la temporada 1924-25 encontramos a la Sociedad de Instrumentos de Viento de París, que ya había actuado en la Sociedad el 12 de mayo de 1903.

Aunque en menor medida, también es importante la presentación de artistas del área germánica: En la temporada 1921-22 el Quinteto Rosé ${ }^{22}$, que ya había visitado la Sociedad en ocho ocasiones anteriores, y Emmy Krüger ${ }^{23}$; En el período 1923-24, el Cuarteto Vocal de Hamburgo, dirigido por John Julia Scheffler, director de masas corales y compositor de música de cámara; En 1924-25 actuó uno de los grupos alemanes de mayor reputación, el Cuarteto Gewandhaus, de Leipzig.

Si bien los intérpretes de otras procedencias son una minoría, es necesario destacar, en la temporada 1923-24, la presencia de Zoltan Székely ${ }^{24}$, procedente de Hungría y acompañante de Bela Bartok desde 1923, así como del Cuarteto Zimmer, de Bruselas, que en 1924 era considerado una de las mejores agrupaciones de cámara del momento ${ }^{25}$.

21. Salazar, Adolfo, "La Vida musical", El Sol, 20 de marzo de 1924. Vera Janacopulos era de procedencia griega, aunque discípula de Georges Enesco y Reja Bauer, (a su vez discípula de Brahms), Jean de Reuzké y Jean Perier. Otras intérpretes en la temporada 1921-22 fueron: Sigrid Hoffmann Onegin, esposa del compositor Engen B. Onegin, aparece señalada en el programa de mano del concierto como una de las primeras "liedersaengerin" del momento. Lola Schlepianoff estudió con Cortot y fue Primer Premio del Conservatorio de París (Ver PÂRIS, Alain, op. cii.).

22. El Cuarteto Rosé, fundado en 1882 por Arnold Rosé, fue considerado como el sucesor de los cuartetos Joachim y Hellmesberger. Arnold Rosé (Iasi, Rumania, 1863-Londres 1946), fue violín solista de la Orquesta Filarmonía de Viena, de Bayreuth y colaborador de la Opera de Viena. Publicó las ediciones de las Sonatas de Bach y de Beethoven, así como el Cuateto op. 18 de este último. Reger le dedicó su Suite al viejo estilo para violín y piano (Ver PÂRIS, Alain, op. cit., p. 558).

23. Emmy Krüger había actuado cinco años en el Teatro Real de Munich y dos mas en el Teatro Imperial de Viena, bajo la dirección de Richard Strauss. Ejecutó su repertorio en la Sociedad Filarmónica el 13 de enero de 1923 y los días 19 y 21 del mismo mes de 1925.

24. Violinista nacido en Kocs (Hungría) en 1903, nacionalizado holandés y, en 1960, estadounidense. Fue discípulo de Kodaly, Hubay y Leo Weiner. Székely, a los 18 años, fue elegido como acompañante por Bartok, realizando su primera actuación a dúo en 1923, actividad que proseguirían durante 15 años. Formó parte del Cuarteto Húngaro, uno de los más importantes de este siglo y creó un nuevo ideal estilístico de la música de Beethoven y Bartok. En la Sociedad Filarmónica de Madrid tuvo como pianista acompañante al también húngaro Janos Baranyi quien, tras ampliar estudios en Berlín con Dohnányi, fue Profesor del Conservatorio de Berlín y de la Academia de Música de Szeged (Ver PÂRIS, Alain, op. cit.).

25. En la temporada 1923-24 destaca, asimismo, la presencia del violinista polaco Bronislaw Hubermann, Lilly HafgrenDinkela, Dagmara Renina y Fernando Ember. Bronislaw Hubermann (Czestochowa, Varsovia, 1882-Lucerna 1947), residió en Viena hasta la llegada del nazismo. Fue miembro del cuarteto Rode, cuyos comienzos efectivos en Europa tuvieron lugar en Berlín, en 1892, cuando estudiaba bajo la dirección de Joseph Joachim, para tener posteriormente un éxito internacional. Lilly Hafgren-Dinkela procedía de Estocolmo, si bien su formación la realizó en Frankfurt y su carrera musical en Italia, Bayreuth, Mannheim y Berlín. Dagmara Renina, aunque de procedencia rusa, se había especializado en óperas italianas, si bien en estos momentos se dedicaba a dar a conocer a los grandes compositores de su país. Fernando Ember, húngaro de nacimiento y formación, se había afincado en España en 1921. 
En conjunto, la Sociedad Filarmónica de Madrid en la temporada 1923-24 contrató trece intérpretes, dos cuartetos y una orquesta ${ }^{26}$, mientras que la Sociedad Sevillana, para la correspondiente a 1922-23, pacta con ocho solistas, dos cuartetos y cuatro orquestas ${ }^{27}$. En esta última Sociedad, la presencia española entre los ejecutantes es mayor, mientras que los foráneos tienen también una procedencia variada, ya que encontramos a músicos rusos (Orquesta Rusa de Balalaikas), ingleses (Cuarteto Londres), franceses (Vera Janacopulos y Lola Schlepianoff) y alemanes (Quinteto Wendling de Stuttgart). Además, los sevillanos tuvieron la oportunidad de asistir a conciertos "históricos". Así podemos denominar los dos conciertos de Wanda Landowska y de Rubinstein, los de este último celebrados los días 16 y 17 de abril de 1923 en el Teatro San Fernando, para los que los socios de la Sociedad tuvieron abonos reducidos ${ }^{28}$.

\section{Clasificación estilística del repertorio ${ }^{29}$}

Respecto a los géneros musicales que integraron los repertorios, en la temporada 1923-24 de la Sociedad Filarmónica de Madrid doce fueron de cámara, seis de solistas y tres de orquestas. Los organizadores, sin embargo, equilibraron las interpretaciones camerísticas, ya que cuatro de ellas fueron de canto y piano, otras tantas de violín y piano y el mismo número de cuartetos ${ }^{30}$. Idéntica proporción se puede observar en períodos anteriores y posteriores: De los ocho programas de mano consultados correspondientes a 1921-22, cuatro fueron de música de cámara, dos de solistas y dos de orquestas ${ }^{31}$; En la temporada 1922-23, de las cinco sesiones examinadas, dos

26. Recitales de canto: Dagmara Renina con Joaquín Turina y Daniel Montorio de pianistas acompañantes (28 de enero de 1924); Lilly Hafgren (canto) y Janos Baranyi (piano) (10 de marzo de 1924). Recitales de canto y piano con partes solísticas para cada uno de ellos: Jeanne Marie Darré (piano), Suzanne Cesbron-Viseur (soprano) y Daniel Montorio (piano de acompañamiento) (24 y 26 de noviembre de 1923). Piano y violín: Zoltan Székely (violín) y Fernando Ember (piano) (10 y 11 de diciembre de 1923); Bronislaw Hubermann (violín) y Siegfried Schultz (21 y 23 de abril). Cuartetos vocales: Cuẩrteto Vocal de Hamburgo, dirigido por John Julia Scheffler (15 y 18 de diciembre de 1923). Cuartetos Instrumentales: Cuarteto Zimmer (11 y 12 de febrero de 1924). Piano solista: José Iturbi (14, 16 y 18 de enero de 1924); Télémaque Lambrino (23 y 25 de febrero de 1924). Violonchelo: Guillermina Suggia (la fecha, en el programa de presentación de la temporada, se halla sin determinar). Orquestas: Orquesta Filarmónica de Madrid, dirigida por B. Pérez Casas, con Télémaque Lambrino (piano) (27 de febrero de 1924); Idem. con Manuel de Falla (címbalo y piano) (28 de marzo de 1924); Idem. con Bronislaw Hubermann (violín) y Siegfried Schultz (26 de abril de 1924).

27. Sociedad Sevillana de Conciertos. Temporada 1922-23. Orquestas: Dos conciertos de óperas cómicas: La Serva Padrona, de Pergolessi y El secreto de Susana, de Wolf Ferrari, interpretadas por Ángeles Ottein, Armando Crabbé, Carlos del Pozo y una orquesta "ad hoc" dirigida por José Anglada (octubre de 1922); Paul Kochanski y Orquesta de veinte profesores dirigida por Falla (23 y 24 de marzo); Orquesta Rusa de Balalaikas, con Helene Sokolskaia y Vladimir Rontchkowsky (10 y 11 de abril); Orquesta Sinfónica, dirigida por Arbós, con la colaboración de la soprano Camino Béjar y de la actriz Herminia Peñaranda (3, 4 y 5 de mayo). Cámara: Dos conciertos del Cuarteto de Londres (noviembre de 1922); Dos conciertos de Vera Janacopulos y Lola Schlepianoff (diciembre de 1922); Dos conciertos del Quinteto Wendling de Stuttgart (enero de 1923); Un concierto de Bildstine y el pianista Zadora (febrero de 1923); Paul Kochanski y Tomás Terán (21 de marzo de 1923). Solistas: Dos conciertos de Wanda Landowska (noviembre de 1922).

28. Así consta en la Memoria de la Sociedad Sevillana de Conciertos de 1923

29. Se ha interpretado el repertorio en base a 162 obras, esto es, prácticamente la mayoría de las escuchadas durante la temporada 1923-24 en la Sociedad Filarmónica de Madrid.

30. Ver nota 26.

31. Recitales de lieder: Sigrid Hoffmann Onegin y H. Frotzler (pianista acompañante) (20 y 22 de febrero de 1922); Quinteto Rosé (15 y 17 de marzo de 1922). Piano solista: Edouard Risler (22 de abril de 1922). Recital de 2 pianos: Magda Tagliaferro y Edouard Risler (26 de abril de 1922). Orquestas: Orquesta Filarmónica, dirigida por Bartolomé Pérez Casas (29 de abril de 1922) y Edouard Risler con la pianista Magda Tagliaferro (1 de mayo de 1922). 
fueron de piano, una de orquesta y dos recitales de canciones $^{32}$; En la comprendida entre 1924-1925, de nueve conciertos, uno fue orquestal y ocho de música de cámara ${ }^{33}$.

La desproporción en favor del género camerístico encuentra en los factores económicos justificación suficiente. Sin embargo, en comparación con lo anotado para lá Filarmónica madrileña, la Sociedad de Conciertos de Sevilla ofreció mayor repertorio orquestal: de los diecinueve conciertos de la temporada 1922-23, nueve pertenecieron a este género, ocho fueron de cámara y dos de solistas $^{34}$.

El primero de los datos que llaman la atención tras estudiar el conjunto de obras escuchadas en la Sociedad Filarmónica de Madrid durante la temporada 1923-24, es su condicionamiento a la procedencia geográfica de las formaciones instrumentales que las ejecutaron. Por ejemplo, el húngaro Zoltan Székely interpretó creaciones de Dvorak y Hubay; el Cuarteto Vocal de Hamburgo incluyó partituras pertenecientes al área alemana de los Siglos XVIII y XIX ${ }^{35}$; Dagmara Renina a autores rusos ${ }^{36}$, mientras que Hubermann y Schultz ofrecieron una obra de Szymanowski ${ }^{37}$.

La segunda cuestión a destacar es que el repertorio se centró fundamentalmente en composiciones del Siglo XIX. El mayor número de las mismas, trece (catorce interpretaciones), corresponden a Liszt ${ }^{38}$. Dentro del área germana destaca Beethoven, con diez partituras ${ }^{39}$, de las cuales tres tienen una fecha de composición anterior a $1803^{40}$, otras tantas corresponden al período "medio" del autor" ${ }^{41}$ mientras que sólo una, el Cuarteto en La menor op. 132 (1824/25) puede

32. Piano solista: José Iturbi (25 y 27 de noviembre de 1922). Orquestas: Orquesta Filarmónica, dirigida por Pérez Casas y José Iturbi como solista (29 de noviembre de 1922). Cámara: Vera Janacopulos (soprano) y Lola Schlepianoff (piano de acompañamiento) (9 y 11 de diciembre de 1922).

33. Orquesta: Orquesta Filarmónica de Madrid, dirigida por Pérez Casas (11 de noviembre de 1924, inauguración de la temporada). Formaciones instrumentales camerísticas: Cuarteto Gewandhaus de Leipzig (19 y 22 de noviembre de 1924); Sociedad de Instrumentos de Viento de París (13 de diciembre de 1924); Sociedad de Música de Cámara de Madrid (24 de enero de 1925); Cuarteto Femenino Rondelé de Bruselas (28 de enero de 1925). Recitales de violín y piano: Antonio Fernández Bordás (violín) y Antonio Lucas Moreno (piano) (16 de diciembre de 1924). Canciones: Emmy Krüger y José Cubiles (Días 19 y 21 de enero de 1925).

34. Ver nota 26.

35. Siglo XVIII: Mozart, Haydn, Kuhlan. Siglo XIX: Beethoven, Brahms, Abt, Schubert, Schumann, Mendelssohn, Woyrsch, Radecke, Scheinpflug, Cornelius, Kirchl y Piber, Thuille, Veit, Debois, Werner. Otros compositores fueron: Palestrina, Gastoldi, De La Hale y Carissimi (15 y 18 de diciembre de 1923).

36. Obras de Mussorgsky, Rimsky, Chaikowsky, Glier, Rachmaninof y Gretchaninof que integraron la primera parte del concierto, mientras que la segunda estuvo dedicada a cantos populares rusos y la tercera a música española (28 de enero de 1924).

37. El resto del repertorio estuvo integrado por obras de: Beethoven (Sonata en La mayor, op. 47); Debussy (Petit Suite, arreglada para piano y violín por H. Hubermann); Lalo (Sinfonía española); C. Franck (Sonata); Bach; Glazunov (Concierto en La menor) (21 y 23 de abril de 1923).

38. Leyenda San Francisco de Paula Caminando sobre las olas; Rapsodia húngara núm. 2 (Suzanne Cesbron-Viseur, Jeanne Marie Darré y Daniel Montorio, 26 de noviembre de 1923); Rapsodia húngara núm. 11; Jeux d'eaux a la villa d'este; Mephisto-Vals (José Iturbi, 14 de enero de 1924); Canzonetta de Salvator Rosa; Rapsodia húngara núm. 12; Sueño de amor; La Campanella (José Iturbi, 16 de enero de 1924); Waldesrauchen; Gnomenreigen; Jeux d'eaux a la villa d'este; Rigoletto-Parafrasis (Télémaque Lambrino, 23 de febrero de 1924); Marcha de Rakoczy (Lilly Hafgren y Janos Baranyi, 20 de marzo de 1924).

39. Sonata en Mi bemol mayor, op. 31, núm. 3 (Jeanne Marie Darré, 26 de noviembre de 1923); Himno a la noche (Cuarteto Vocal de Hamburgo, 15 de diciembre); Gloria a Dios en la naturaleza (Cuarteto Vocal de Hamburgo, 18 de diciembre); Sonata en Sol mayor, op. 14, núm. 2 (José Iturbi, 16 de enero); Cuarteto en Mi menor, op. 59, núm. 2 (Cuarteto Zimmer, 11 de febrero); Cuarteto en La menor, op. 132 (Cuarteto Zimmer, 12 de febrero); Sonata en Do sostenido menor, op. 27, núm. 2 (Télémaque Lambrino, 23 de febrero); Rondo a Capriccio en Sol mayor, opus 129; Andante favori en Fa mayor (Janos Baranyi, 10 de marzo); Sonata en La mayor, op. 47 (Bronislaw Hubermann y Siegfried Schultz, 21 de abril).

40. Sonata op. 14, núm. 2; Sonata op. 27, núm. 2 y Sonata op. 31, núm. 3.

41. Sonata op. 47; Cuarteto en Mi menor, op. 59, núm. 2 y Andante Favori en Fa Mayor. 
adscribirse a su última etapa. El romanticismo alemán está presente en siete obras de Schubert, cinco de Schumann, cuatro de Brahms y Mendelssohn y una de Wagner y R. Strauss ${ }^{42}$.

A los autores anteriores hemos de añadir otros como Chopin, del que se interpretan siete composiciones, y Berlioz, escuchado sólo en una ocasión ${ }^{43}$. La presencia de músicos franceses aumenta con partituras de Gabriel Fauré, César Franck, Saint-Saëns y Vincent D'Indy ${ }^{44}$.

El tercer aspecto importante es que, después de Liszt, el autor de cuyo repertorio se interpretan mas obras, trece, es Debussy, siendo las suyas las más novedosas desde el punto de vista estilístico de las que configuraron la temporada de la Sociedad Filarmónica de Madrid, junto a la única ejecución de Rispetti e Strambotti, de Malipiero, y dos partituras de Ravel ${ }^{45}$. Tanto este último como Debussy se hallan asimismo presentes en los conciertos que la Orquesta Bética ofrece en la Sociedad de Conciertos de Sevilla ${ }^{46}$.

El cuarto punto interesante es la reducida representación de autores del período clásico, ya que sólo se escuchan tres composiciones de Haydn y otras tantas de Mozart ${ }^{47}$, siendo aún menos las procedentes de la primera parte del XVIII ${ }^{48}$. Por el contrario, en la Sociedad Sevillana de Conciertos, ya en 1922 y 1923 se interpretó este repertorio ${ }^{49}$, incrementándose a partir de 1924 puesto que la Orquesta Bética de Cámara incluía obras de Haydn, Mozart y Scarlatti ${ }^{50}$. Lo mismo

42. Schubert: Impromptu en Si bemol, op. 142, núm. 3; Momento musical en Fa menor, op. 94, núm. 3 (José Iturbi, 16 de enero); El Tilo (Cuarteto Vocal de Hamburgo, 15 de diciembre); Rosa Silvestre (Cuarteto Vocal de Hamburgo, 15 de diciembre); El Gondolero (Cuarteto Vocal de Hamburgo, 15 de diciembre); Salmo 23 (Cuarteto Vocal de Hamburgo, 18 de diciembre); Schubert-Liszt: "Valse-Caprice" (Soirées de Vienne, núm. 6) (Jeanne Marie Darré, 24 de noviembre). Schumann: Sonata en Sol menor, op. 22; Carnaval, op. 9; Escenas de niños, op. 16; Estudios sinfónicos, op. 13 (Télémaque Lambrino, 25 de febrero); El Lago dormido (Cuarteto Vocal de Hamburgo, 15 de diciembre). Brahms: Canción de Cuna (Cuarteto Vocal de Hamburgo, 15 de diciembre); Serenata; Domingo (Cuarteto Vocal de Hamburgo, 18 de diciembre); Variaciones sobre un tema de Paganini (José Iturbi, 16 de enero). Mendelssohn: Canto de pastor (Cuarteto Vocal de Hamburgo, 15 de diciembre); La Caza; La Hilandera; Rondó caprichoso, op. 14 (José Iturbi, 16 de enero). Wagner: Schmerzen; Träume (Lilly Hafgren y Janos Baranyi, 10 de marzo). Strauss: Burleske en Re menor (Télémaque Lambrino con la Orquesta Filarmónica dirigida por B. Pérez Casas, 27 de febrero).

43. Chopin: Estudio en Mi mayor, op. 10, núm. 3; Estudio en Do menor, op. 10, núm. 12; Vals en La bemol mayor, op. 42 (Jeanne Marie Darré, 24 de noviembre); Vals en Sol bemol, op. 70, núm. 1; Polonesa en La bemol, op. 53 (José Iturbi, 14 de enero); Sonata en Si menor, op. 58; Concerto en Fa menor, op. 21 (Télémaque Lambrino, 23 de febrero y junto a la Orquesta Filarmónica de Madrid el 27 de febrero). Berlioz: Aria de la Damnation de Faust (Suzanne Cesbron-Viseur, 24 de noviembre).

44. Fauré: Automne; Clair de Lune; Soir. Franck: La Procession (Jeanne Marie Darré, 26 de noviembre); Preludio, Fuga y Variación (José Iturbi, 14 de enero). Saint-Saëns: Bourré, op. 135, núm. 4 (Jeanne Marie Darré, 24 de noviembre). D’Indy: Cuarteto en Mi mayor, op. 45 (Cuarteto Zimmer, 11 de febrero).

45. Debussy: Aria de L'Enfant prodigue; Romance; C'Est l'Extase Langoureuse; Il Pleure dans mon coeur (Suzanne Cesbron-Viseur, 24 de noviembre); Toccata; Nocturno (Jeanne Marie Darré, 24 de noviembre); Arabesque núm. 1; Arabesque núm. 2; L'Isle Joyeuse (José Iturbi, 14 de enero); Sarabanda; Reflets dans l'eau (Télémaque Lambrino, 23 de febrero); Petit suite (arreglo para violín y piano de B. Hubermann, 21 de abril). Ravel: Piéce (Zoltan Székely y Fernando Ember, 10 de diciembre); Pavane pour une infante defunte (José Iturbi, 14 de enero). Malipiero: Rispetti e Strombotti (Cuarteto Zimmer, 12 de febrero).

46. Ravel: Mi madre la Oca; Debussy: Preludio a la Siesta de un fauno (10 de diciembre de 1924. Concierto núm. 83). Debussy: Petit Suite; Ravel: Pavana para una Infanta difunta. Georges Migot: Le Paravent de Lague aux 5 linajes (20 de noviembre de 1925. Concierto 102). Ravel: "Minuetto" y "Rigaudon" de la Suite Le Tombeau de Couperin (21 de noviembre de 1925. Concierto 103).

47. Haydn: Serenata (Cuarteto Vocal de Hamburgo, 15 de diciembre); Sonata en Mi menor (José Iturbi, 14 de enero); Recitado y Aria de Die Jahreszeiten (Lilly Hafgren y Janos Baranyi, 10 de marzo). Mozart: Aria de La Flauta Mágica (Cuarteto Vocal de Hamburgo, 15 de diciembre); Cuarteto en Si bemol mayor, K. 458 (Cuarteto Zimmer, 11 de febrero); Sinfonía en Sol menor, $K .550$ (Orquesta Filarmónica de Madrid, 28 de marzo).

48. J.S. Bach: Concerto in gusto italiano. D. Scarlatti: Sonata en Mi mayor. Sonata en Do mayor (José Iturbi, 18 de enero).

49. Pergolessi: La Serva Padrona. Wolf Ferrari: El secreto de Susana (octubre de 1922). Mozart: Sonata en Si bemol Mayor. J.S. Bach: Preludio y Adagio (Paul Kochanski. Marzo de 1923).

50. Haydn: Sinfonía en Mi bemol (10 de diciembre de 1924. Concierto núm. 83). Mozart: Sinfonía en Sol menor. Scarlatti: "Allegro", “Adagio" y "Allegrísimo" (12 de diciembre de 1924. Concierto núm. 84). Mozart: Las bodas de Fígaro. Haydn: 
podemos decir para la temporada 1925-26, en cuyo mes de noviembre dicha Orquesta ofreció cuatro conciertos en el Teatro San Fernando con partituras clásicas y barrocas ${ }^{51}$ que superaron al conjunto integrado por composiciones del romanticismo alemán, francés e italiano ${ }^{52}$.

En quinto lugar, es necesario destacar la escasa presencia de músicos nacionalistas en la Filarmónica de Madrid, como Dvorak, siendo los más numerosos los pertenecientes a la escuela rusa $^{53}$. Este dato contrasta con otros programas también madrileños, como los de la Orquesta Filarmónica de Madrid, que en la Sociedad Filarmónica interpretó repertorio alemán y español, pero que llevó obras de Rimsky, Borodin y Mussorgsky por provincias ${ }^{54}$. La misma formación, en el Teatro Real, el día 25 de marzo de 1924, ofreció un concierto denominado "Gran Concierto Ruso", bajo la dirección de Strawinsky y Pérez Casas ${ }^{55}$. El mismo hecho se puede observar en otras formaciones, como la recién nacida Orquesta Bética que interpretó composiciones de autores rusos por todo el país, especialmente de Glinka y Strawinsky ${ }^{56}$. No podemos olvidar tampoco la presencia en la Sociedad de Conciertos de Sevilla de la Orquesta Rusa de Balalaikas en abril de 1923, con obras populares, ni que los socios de esta Sociedad conocerían repertorio de autores rusos a través de los conciertos que Rubinstein ofreció en Sevilla ininterrumpidamente desde 1922 a $1926^{57}$.

En definitiva, de los 82 compositores presentes en la temporada 1923-24 de la Sociedad Filarmónica $^{58}$, veintidós de ellos se encuadran en el romanticismo alemán; otros once pertenecen

Sinfonía en Re mayor núm. 14 (20 de noviembre de 1925). J.S. Bach: Segundo Concierto en Fa (21 de noviembre de 1925). J.S. Bach: Partita en Do menor. Mozart: Sonata. Haendel: Concerto en Si bemol mayor (cadencia improvisada por W. Landowska). Telemann: Bourrèe. F. Couperin: Les vergers fleuris. Cl. Daquin: Le coucou. D. Scarlatti: La Chasse (Wanda Landowska. 25 de noviembre de 1925). Bach: Preludio en Do mayor. Haendel: Le forgeron harmonieux. J.S. Bach: Capriccio sur le départ son frére bien aimé. Haydn: Sonata en Do mayor. Mozart: Andante amoroso. Clementi: La pantoufle. Rameau: Les sauvages. La follette. F. Couperin: Les vergers fleuris. W. Landowska: Bourrèe d'Auvergne (Wanda Landowska. 27 de noviembre de 1925).

51. Mozart: Las bodas de Fígaro. Sonata. Andante amoroso. Haydn: Sinfonía en Re mayor núm. 14. Sonata en Do mayor. D. Scarlatti: La chasse. J.S. Bach: Segundo Concierto en Fa. Partita en Do menor. Preludio en Do mayor. Capriccio sur le départ son frére bien aimé. Haendel: Concerto en Si bemol mayor (cadencia improvisada por W. Landowska), Le forgeron harmonieux. Telemann: (Bourrè). F. Couperin: Les vergers fleuris (20, 21, 25 y 27 de noviembre de 1925. Conciertos 102, 103, 104 y 105 . Orquesta Bética dirigida por Ernesto Halffter).

52. Wagner: Idilio de Sigfrido (Orquesta Bética. 12 de diciembre de 1924). Rossini: El Barbero de Sevilla (revisión orquestal de Falla) (20 de noviembre de 1925). Fauré: Suite de Pelleas et Mélisande. Wagner: Idilio de Sigfrido (21 de noviembre de 1925). Además, los siguientes autores habían sido escuchados los meses anteriores: Chopin: Cinco pequeñas piezas. Nocturno. Beethoven: Sonata op. 47 (21 de marzo de 1923). Beethoven: Sonata en Sol mayor op. 30, núm. 3. Wagner-Wilhelmy: Preishlied (23 de marzo de 1923). Liszt: En el bosque. Chopin: Polonesa op. 53. Paganini-Kochanski: La Campanela (24 de marzo de 1923).

53. Dvorak: Concerto (Zoltan Szekely y Fernando Ember. 10 de diciembre de 1924). Cuarteto en Sol mayor, op. I06 (Cuarteto Zimmer. 12 de febrero). Rimsky-Korsakof: La Alondra. Mussorgsky: Canto hebreo. Gliere: No quiero amarte. Gretchaninoff: Canción de cuna. Rachmaninof: Lilas. Lluvias primaverales. Chaikowsky: Canto del Volga (Dagmara Renina, 28 de enero). Concerto en Si bemol menor, op. 23 (Télémaque Lambrino, 27 de febrero).

54. 2 y 3 de junio de 1924 en el Teatro Isabel la Católica de Granada.

55. Se estrenaron en Madrid la Suite del Pájaro de Fuego "para orquesta media" y la Suite Pulcinella, además de la interpretación de Sheherazada y Capricho español de Rimsky y La Princesa lejana de Tscherepnin.

56. En su concierto de presentación el 11 de junio de 1924, en el Teatro Llorens de Sevilla, introdujo en el programa Suite Pulcinella, de Strawinsky, y Kamariskä̈a, de Glinka. Esta última obra la interpretó, entre otras muchas ocasiones, el 12 de diciembre de 1924 en la Sociedad Sevillana de Conciertos; el 30 de enero de 1925 en el Teatro San Fernando de Sevilla, etc.

57. Prokofief: Marcha. Visión fugitiva. Sugestión Diabólica (17 de abril de 1922). Strawinsky: Petrouchka (29 de abril de 1925, Sociedad Sevillana de Conciertos) (ver Martin MOREno, A., "Rubinstein y Andalucía" en VARIOS, Rubinstein y España, Madrid, Fundación Isaac Albéniz, 1987, pp. 116-119.

58. El repertorio de la Sociedad Filarmónica de Madrid en la temporada 1923-24 se completa con autores que, como Henneberg (Lieders populares suecos: Canto a la primavera. Espera inútil), Rachmaninof (Lilas. Lluvias primaverales), Scott (Melodie et danse nègre. Tierra del loto) y Scriabin (Poema, op. 32, núm. 1. Mazurca, op. 40, núm. 2), están representados con 
a la escuela francesa del XIX y principios del XX; nueve son autores del Siglo XVIII de Alemania, Italia, España y Francia; doce son compositores nacionalistas, principalmente rusos; nueve son españoles del XIX y XX, y seis se sitúan cronológicamente en siglos anteriores.

El análisis anterior es similar al de otras temporadas de la Sociedad Filarmónica de Madrid $^{59}$, como la de 1924-25, en que de 69 obras correspondientes a los nueve primeros conciertos, 40 son del romanticismo alemán, siete del repertorio alemán del Siglo XVIII, dos del italiano del mismo siglo, tres del nacionalismo ruso y cuatro de la escuela francesa de finales del XIX y principios del $\mathrm{XX}^{60}$.

\section{Presencia de la música española}

No cabe duda que, tanto en la temporada 1923-24 de la Sociedad Filarmónica de Madrid como en la Sociedad de Conciertos de Sevilla en 1922-23, los momentos estelares fueron las audiciones de El Retablo. El 23 de marzo de 1923 se estrenó sin muñecos en Sevilla ${ }^{61}$ y el 28 de marzo de 1924 en el Teatro de la Comedia, por primera vez en la capital de España. La Orquesta de Cámara de Sevilla, dirigida por Ernesto Halffter y Manuel de Falla, con la cooperación de la soprano Mercè Plantada, ofrecerían, el 7 de febrero de 1925, el estreno de la misma obra en Barcelona, junto a El Amor Brujo.

Tanto en Madrid como en Sevilla, El Retablo se ofreció sin representación escénica. En los programas de mano se presentó un extenso resumen de la obra, posiblemente por exigencia del mismo Falla quien, según indica Federico Sopeña ${ }^{62}$, había manifestado tal deseo con motivo de su estreno, antes citado, en Sevilla, en 1923.

Dejando de lado estos acontecimientos históricos, la presencia de la música española en el repertorio de la Filarmónica de Madrid fue escasa en relación con la de procedencia extranjera.

dos o tres obras, además de un reducido número de canciones populares y un abultado grupo de autores con una sola obra (Abt, Agus, Aubert, Carissimi, Cornelius, Chausson, D’Ambrosio, Daquin, De la Hale, Debois, Dohnanyi, Duparc, Gastoldi, Glazunov, Gluck-Sgambati, Gretry, Erik Hafgren, Hildach, J. Hubay, Hüe, Kirchl, Kuhlau, Lalo, Palestrina, Pasquini, Pftizner, Piber, Poldowsky, Radecke, Scharwenka, Scheinpflug, Scott, Sinding, R. Strauss, Székely, Szymanowski, Thuille, Veit, Veracini, Vieuxtemps, Weber, Werner, Wolf, Woyrsch).

59. Temporada 1921-22. Sigrid Hoffmann Onegin: Obras de Schubert, Schumann, Brahms, Pergolessi, Lotti, Caldara, Paisiello, Martini, Bizet, Debussy, Joncieres y Onegin. Quinteto Rosé: Obras de Brahms, Schumann, Schubert, Franck, Glazunov y Borodin. Edouard Risler: Obras de Beethoven. Edouard Risler y Magda Tagliaferro: Obras de Mozart, Schumann, Liszt, Chabrier y Saint-Saëns. Orquesta Filarmónica de Madrid: Obras de Mozart, Beethoven y Grieg. Temporada 1922-23. José Iturbi: Obras de Mozart, Beethoven, Ravel, Balakiref, Debussy, Chopin y Liszt. Vera Janacopulos: Obras de Scarlatti, Pergolessi, Martini, Mozart, Mussorgsky, Duparc, Fauré, A. Roussel, Schubert, Rimsky, Rachmaninof, Prokofief y Falla.

60. Temporada 1924-25. Orquesta Filarmónica: Obras de Haendel, Mozart, Wagner, Debussy. Cuarteto Gewandhaus: Obras de Mozart, Chaikowsky, Beethoven, Schumann y Weismann. Sociedad de Instrumentos de Viento de París: Obras de Mozart, Haendel, Roussel, Rimsky-Korsakof, D’Indy, Jongen. Antonio Fernández Bordás y Lucas Moreno: Obras de Nardini, Beethoven, Bach, Schubert, Kreisler, Svendsen, Tartini y Sarasate. Emmy Krüger y José Cubiles: Obras de Schubert, Schumann, Wolf, Franck, Brahms, Löwe. Sociedad de Música de Cámara de Madrid (Instrumentos de Viento): Obras de Saint-Saëns, Albisi, Huguenin, Beethoven, Bach, Thuille. Cuarteto Femenino Redelé: Obras de Schubert, Dvorak, Borodin.

61. Con la colaboración del violinista Paul Kochanski y el pianista Tomás Terán se interpretaron, en la misma sesión, las siguientes obras: Beethoven: Sonata en Sol mayor, op. 30, núm. 3. Granados: Allegro de concierto. Albéniz: Navarra y Triana. Reay Colazo: Fado. Falla: Danza del amor brujo. Wagner/Wilhelmy: Preishlied. Kreisler: Gitana. Rimsky: Canto Indio. Sarasate: Zapateado.

62. SopeÑa, Federico, Vida y obra de Falla, Madrid, Turner, 1988, p. 130

[10] 
Entre los intérpretes europeos, sólo Zoltan Székely y Dagmara Renina la incluyeron en sus conciertos, y toda ella, excepto dos obras de Scarlatti, estuvo centrada en autores de los últimos años del Siglo XIX y principios del XX, cuyas partituras se escuchaban por vez primera en dicha Sociedad $^{63}$.

El número de obras españolas ejecutadas parece especialmente precario si lo comparamos con el de diversos conciertos de la misma época ofrecidos en otros lugares de España, especialmente en Barcelona, donde es posible encontrar sesiones que, como el recital de piano de María Carratalá, titulado "Primera manifestació de la moderna escola musical catalana" ${ }^{\text {, }}$, dedicaron un lugar significativo a la creación nacional.

Por otra parte, la inclusión del repertorio español y su consiguiente conocimiento por parte del público de la Sociedad Filarmónica de Madrid se halla absolutamente unida a los intérpretes nacionales como José Iturbi ${ }^{65}$ y Pilar Bayona ${ }^{66}$, hecho que también se pone de manifiesto en los conciertos que Andrés Segovia ${ }^{67}$, Joaquín Nin ${ }^{68}$ o el Quinteto Hispania ${ }^{69}$ ofrecieron en diversas sociedades del país. Es justamente el espacio dedicado a los compositores más jóvenes el que Adolfo Salazar señala singularmente al elaborar la crítica musical del año 1923:

"El hecho significativo del año transcurrido es precisamente esa afirmación de fuerzas jóvenes que vienen a continuar la historia, a añadir un nuevo capítulo a los ya conocidos. En varios terrenos, y siguiendo distintas tendencias, se plantea, sin duda, ese relevo de personas; pero lo importante es, a juicio mío, la renovación de criterios."

63. Manén: Canción (Zoltan Székely y Fernando Ember. 10 de diciembre de 1923). Granados/Kreisler: Danza española, primera audición (Mismos intérpretes. 11 de diciembre). Infante: Gitanerías, primera audición (José Iturbi, 14 de enero de 1924). D. Scarlatti: Sonata en Mi mayor, primera audición y Sonata en Do mayor. Granados: El Pelele, primera audición. Albéniz: Jerez y Navarra, primeras audiciones. El Puerto. Triana (José Iturbi, 18 de enero de 1924). Vives: Madre, la mi madre. Albéniz: Dos rimas de Becquer. Moreno Torroba: Copla de Antaño. Falla: Nana. J. Gómez: Canción árabe. Turina: Poema. (Dagmara Renina y Joaquín Turina, 28 de enero de 1924. Todas las obras de este programa, excepto Nana de Falla, son primicias en la Sociedad). Falla: El Retablo de Maese Pedro. Noches en los jardines de España (Orquesta Filarmónica dirigida por Pérez Casas. Manuel de Falla interpreta el címbalo y piano. 28 de marzo de 1924. Primera audición en Madrid de El Retablo).

64. Obras de Lluis Millet, A. Noguera, F. Pedrell, F. Mompou, R. Gerhard, E. Morera, I. Albéniz, J. Pahissa y F. Pedrell (17 de mayo de 1925). Otros ejemplos son: En el Gran Teatro del Liceu, en mayo de 1924, la Orquesta Pau Casals ofreció dos sesiones extraordinarias con los programas que se ejecutarían en París en la "Grande Sesion d'Art de la VIII I Olympiade", con obras de Manén, Garreta (15 de mayo), Falla (La vida breve), Granados (Goyescas) y Albéniz (Catalonia) (19 de mayo). El 26 de febrero de 1925 , el Trio Barcelona (Ricard Vives, Marián Perelló y J. Pere Masés) ejecutaron obras de Bretón, Perelló y Granados. Los tres conciertos extraordinarios de Cuaresma de 1925 que ofrecieron en el Palau de la Música María Barrientos y el pianista Tomás Terán, incluyeron obras de P. San Sebastián, Blas de Laserna, Granados, Turina, Amadeo Vives, Falla (Siete Canciones populares), Alió y Nicolau.

65. Ravel: Pavane pour une infante défunte. Arabesque núm. 1. Debussy: Arabesque núm. 2. L'Isle Joyeuse. Infante: Gitanerías (Sociedad Filarmónica de Madrid, 14 de enero de 1924). Debussy: Preludio, Sarabanda y Toccata. Albéniz: El Corpus en Sevilla. El Puerto. Granados: Danza en Sol mayor. Infante: Sevillana y El Vito (Sociedad Filarmónica de Madrid, 27 de noviembre de 1922).

66. Debussy: Las colinas de Anacapri. Estudio para los ocho dedos. Ravel: La alborada del gracioso. Falla: Danza de El Amor Brujo. Ernesto Halffter: Lullaby, P. San Sebastián (Sociedad Filarmónica de Madrid, enero de 1923) (ver SalazAR, Adolfo, "La vida musical", El Sol, 25 de enero de 1923.

67. Obras de Sor, Coste, Albéniz y Granados (Hotel Ritz, 21 de mayo de 1917). Sors, Ponce, Tárrega, Turina, Falla (Homenaje a Debussy), Torroba, Albéniz y F. de Lacerda (Sociedad Filarmónica de Vigo, 29 y 30 de diciembre de 1924).

68. Obras de Couperin, Rameau, Scarlatti, Soler, Bach, Mozart, Chopin, Granados, Debussy, Mussorgsky y Grieg (Sociedad Filarmónica de Madrid, 23 y 25 de mayo de 1917).

69. Obras de Turina y Esplá (Asociación de Cultura Musical de Madrid, enero de 1923). Lamote de Grignon, Franco, Bacarisse, Lloret, Mantecón, Halffter y Salazar (Sala Aeolian de Madrid, mayo y junio de 1923) (ver SalazAR, Adolfo, "El año musical", El Sol, 1 de enero de 1924).

70. Salazar, Adolfo, "El Año Musical”, El Sol, 1 de enero de 1924. 
Una última cuestión importante es que la música española, tanto actual como histórica, está vinculada en los programas a la creación contemporánea europea. Así aparece en los conciertos ya mencionados de José Iturbi y Pilar Bayona ${ }^{71}$ y de ciertos intérpretes extranjeros, como Vera Janacopulos $^{72}$. En este sentido, la contribución de la Orquesta Bética parece especialmente importante ${ }^{73}$, ya que su finalidad era, como señala Salazar con motivo de su presentación, la de ofrecer el repertorio clásico y contemporáneo:

"la Orquesta Bética está fundada para dedicar preferentemente su actividad a la interpretación de la música de los primeros clásicos y de los últimos autores contemporáneos —últimos y primeros a la vez-." 74

\section{Novedad del repertorio}

En epígrafes anteriores hemos señalado la superioridad del repertorio romántico en la Sociedad Filarmónica de Madrid, dato que, sin embargo ha de completarse con otro bien significativo: la mayoría de estas obras supusieron una primicia en la Sociedad o, en su caso, en Madrid $^{75}$. Así, en la temporada 1923-24 el Cuarteto Vocal de Hamburgo ejecutó por vez primera en la Sociedad obras de Beethoven, Brahms, Mozart, Schubert, Schumann, Haydn, Mendelssohn y Carissimi; Télémaque Lambrino aportó como novedades obras de Chopin, Chaikowsky y Strauss, mientras que Lilly Hafgren y Janos Baranyi contribuyeron al conocimiento de Haydn, Beethoven, Liszt, Pftizner, etc ${ }^{76}$.

La novedad del repertorio se hace aún más evidente en intérpretes como Dagmara Renina, cuyos recitales sólo incluyeron dos obras ya conocidas por el público ${ }^{77}$, y Zoltan Székely y

71. Ver notas 64 y 65 .

72. Prokofief: Romanza sin palabras. Falla: El Paño moruno. Seguidillas murcianas. Asturiana. Jota. Nana. Canción. Polo (Sociedad Filarmónica de Madrid, 11 de diciembre de 1922).

73. Algunos ejemplos de sus programas son: Falla: Amor Brujo, El Retablo (estreno en Barcelona), Psiquis y El Sombrero de tres picos. Mozart: Sinfonía en Sol menor. Rimsky: Sinfonieta en La menor sobre temas rusos. Glinka: Kamarinskaïa (Associació de Música "Da Cámera" de Barcelona. 7 y 9 de febrero de 1925). El mismo repertorio el 30 de enero de 1925 en el Teatro San Fernando de Sevilla. Falla: El Amor Brujo. Glinka: Kamarinskaïa. Scarlatti/Roland Manuel: Trois Pieces. A. Salazar: Dos Preludios. R. Halffter: Berceuse. E. Halffter: Marcha Grotesca. Strawinsky: Suite Pulcinella (Teatro Llorens de Sevilla. 11 de junio de 1924). Ravel: Mi madre la Oca. Debussy: Preludio a la siesta de un fauno. Glinka: Kamarinskä̈a. Falla: El Sombrero de Tres picos y El Retablo (Asociación de Conciertos de Reus. 11 de febrero de 1925). Idéntico programa en la Sociedad Filarmónica de Valencia (Teatro Principal. 2 y 4 de febrero de 1925. Estreno de El Retablo en dicha Sociedad). Falla: El Amor Brujo (Sociedad Sevillana de Conciertos. 10 de diciembre de 1924). El sombrero de tres picos (Sociedad Sevillana de Conciertos. 12 de diciembre de 1924 y 21 de noviembre de 1925).

74. Salazar, Adolfo, "La Vida Musical, El Sol, s.d. 1924. Recoge buena parte del texto redactado por Manuel de Falla para dicha ocasión (FAlla, Manuel de, "Orquesta Bética de Cámara", El Correo de Andalucía, 6 de junio de 1924).

75. Los programas de mano indican estos datos mediante asteriscos.

76. Las aportaciones novedosas del Cuarteto Zimmer se refieren a un ámbito más variado, ya que estrenaron el Cuarteto en Mi mayor, op. 45 de D'Indy, el Cuarteto en Sol mayor, op. 106 de Dvorak, y Rispetti e Strambotti de Malipiero, junto a obras ya escuchadas de Mozart y Beethoven. Jeanne Marie Darré y Suzanne Cesbron-Viseur ejecutaron como novedades obras de Pasquini, Gluck/Sgambati, Gretry, Berlioz, Chopin, Debussy (Aria de L'Enfant prodigue, Nocturno), Hüe, Aubert (Poémes arabes) y Fauré, además de otras ya conocidas como Toccata de Debussy, estudios de Chopin, sonatas de Beethoven y obras de Franck y Liszt. 77. Gretchaninof: Canción de Cuna. Falla: Nana. 
Fernando Ember quienes, con la excepción de Ballade et Polonaise del violinista belga Henri Vieuxtemps, ofrecieron exclusivamente primeras audiciones.

Como se puede observar, las primicias suponen una gran mayoría, por lo que podemos concluir que dichas obras, a pesar de que principalmente pertenecen al período romántico, suponen un descubrimiento que se extiende, aunque en menor proporción, a la música contemporánea tanto española como europea. Este hecho puede hacerse extensivo, además, al repertorio escuchado en la Filarmónica madrileña en temporadas anteriores y posteriores.

Respecto a la Sociedad de Conciertos de Sevilla ${ }^{78}$, es de nuevo la presencia de la Orquesta Bética la que, a partir de 1923, posibilitó el acceso del público sevillano a un repertorio innovador, a lo que es necesario sumar conciertos tan emblemáticos como los cuatro de la temporada 1925-26 a cargo de esta última formación y Wanda Landowska, así como los dos de Rubinstein. Este talante innovador se halla también en la Memoria de la temporada 1922-23 de la Sociedad Sevillana de Conciertos, en la que podemos leer la siguiente declaración de principios que singulariza sus finalidades:

\footnotetext{
"El desarrollo progresivo de nuestra cultura musical nos pone, por otra parte, en condiciones de exigir elementos superiores, y en este plan, hemos de procurar que ante nosotros desfile todo aquello que constituye novedad artística o cuanto en el mundo musical revista caracteres resonantes.",79
}

\section{Conclusiones}

1. Las temporadas correspondientes a los años 1921 a 1925 de la Sociedad Filarmónica de Madrid estuvieron integradas por una media de veinte conciertos anuales, cifra similar a la de la Sociedad Sevillana de Conciertos en el período 1922-23.

2. La contratación de artistas se realizaba dentro de giras, con intérpretes que ejecutaban el mismo repertorio en distintas sociedades por todo el país, con frecuencia de manera recurrente y para dos sesiones consecutivas.

3. En la Sociedad Filarmónica de Madrid, en la temporada 1923-24, la presencia de intérpretes españoles fue muy escasa, reduciéndose a la de José Iturbi, Daniel Montorio y la Orquesta Sinfónica. Entre los extranjeros, destacan los de procedencia francesa, tanto por su número como por la frecuencia de sus actuaciones.

4. El 57\% de las sesiones de la Filarmónica de Madrid en la temporada 1923-24 fueron de cámara, $28,5 \%$ de solistas y el $14 \%$ de orquestas, proporciones similares a las de otras

78. Otros autores presentes en la Sociedad de Conciertos, además de los mencionados hasta aquí, son: Pugnani: Preludio y allegro. Rimsky-Korsakof: Himno al Sol. Canto Indio. Winieawsky: Carnaval ruso. Reay Colazo: Fado. Kreisler: Gitana. Grieg: Sonata en Do menor, op. 45. Daquin: El Cucú. Desplanes: Intrada. Dvorak: Danza eslava.

79. Memoria presentada por la Junta directiva a los Socios en la Junta General Ordinaria de 25 de junio de 1923 , p. 11. Otras de las finalidades de la Sociedad aparecen también mencionadas en dicha Memoria, como la creación de premios e incentivos para alumnas estudiantes de piano y solfeo en la Sociedad Económica de Amigos del País, su relación con otras Sociedades de Conciertos de Cádiz, Huelva, Córdoba y Jaén, así como con diversas asociaciones de carácter internacional. 
temporadas. La Sociedad Sevillana de Conciertos, en la temporada 1922-23, equilibró la proporción: $47,4 \%$ de orquestas, $42,1 \%$ de cámara y $10,5 \%$ de solistas.

5. El repertorio escuchado en la Filarmónica de Madrid durante la temporada 1923-24 estuvo condicionado a la procedencia geográfica de los intérpretes que lo ejecutaron. Cronológicamente, se centró en composiciones del Siglo XIX, destacando las correspondientes al romanticismo alemán y a autores franceses de la segunda parte del mismo Siglo. No obstante, fue Debussy, después de Liszt, el autor más interpretado y su obra, en conjunto, la más novedosa desde el punto de vista estilístico. Aunque en menor proporción, también se presentaron obras del Siglo XVIII y de autores nacionalistas rusos, ampliamente incluidas en los conciertos de la Sociedad Sevillana en la temporada 1922-23.

6. La presencia de la música española en el repertorio de la Filarmónica de Madrid durante la temporada 1923-24 fue escasa en relación con la de procedencia extranjera. Los compositores más escuchados fueron Albéniz, Granados y Falla, cuyas obras fueron incluidas en los conciertos de intérpretes españoles que, además, introdujeron autores del Siglo XVIII español y música contemporánea europea.

7. La gran mayoría del repertorio interpretado en la temporada 1923-24 en la Filarmónica se ofreció por vez primera al público de la Sociedad y a la misma capital de España. 